\title{
BMJ Open Symptom burden, Metabolic profile, Ultrasound findings, Rhythm, neurohormonal activation, haemodynamics and health-related quality of life in patients with atrial Fibrillation (SMURF): a protocol for an observational study with a randomised interventional component
}

\author{
Emmanouil Charitakis, ${ }^{1}$ Ulla Walfridsson, ${ }^{1}$ Fredrik Nyström, ${ }^{2}$ Eva Nylander, ${ }^{3}$ \\ Anna Strömberg, ${ }^{1}$ Urban Alehagen, ${ }^{1}$ Håkan Walfridsson ${ }^{1}$
}

To cite: Charitakis $\mathrm{E}$, Walfridsson U, Nyström F, et al. Symptom burden, Metabolic profile, Ultrasound findings, Rhythm, neurohormonal activation, haemodynamics and healthrelated quality of life in patients with atrial Fibrillation (SMURF): a protocol for an observational study with a randomised interventional component. BMJ Open 2015:5:e008723.

doi:10.1136/bmjopen-2015008723

- Prepublication history for this paper is available online. To view these files please visit the journal online (http://dx.doi.org/10.1136/ bmjopen-2015-008723).

Received 13 May 2015 Revised 16 September 2015 Accepted 12 October 2015

CrossMark

For numbered affiliations see end of article.

\section{Correspondence to} Dr Håkan Walfridsson; hakan.walfridsson@ regionostergotland.se

\section{ABSTRACT}

Introduction: Atrial fibrillation (AF) is the most common cardiac arrhythmia, with an estimated prevalence of $1.5-2 \%$. It is an independent risk factor for ischaemic stroke and is estimated to cause about $20-25 \%$ of all stroke cases. AF has a great impact on health-related quality of life (HRQoL); however, one unresolved issue related to $A F$ is the wide variation in its symptoms.

Methods and analysis: The symptom burden, metabolic profile, ultrasound findings, rhythm, neurohormonal activation, haemodynamics and $\mathrm{HRQ}$ oL in patients with $\mathrm{AF}$ (Symptom burden, Metabolic profile, Ultrasound findings, Rhythm, neurohormonal activation, haemodynamics and healthrelated quality of life in patients with atrial Fibrillation, SMURF) study is a prospective observational, cohort study, with a randomised interventional part. The aim of the study is to investigate, in patients with AF, the relationship between symptom burden and metabolic aspects, atrial function and different neurohormones, and the effect of radiofrequency ablation (RFA). The interventional part of the study will give an insight into the neurohormonal and intracardiac pressure changes directly after initiation of AF. Consecutive patients with symptomatic AF accepted for treatment with RFA for the first time at Linköping University Hospital are eligible for participation. The enrolment started in January 2012, and a total of 200 patients are to be included into the study, with 45 of them being enrolled into the interventional study with initiation of AF. The sample size of the interventional study is based on a small pilot study with 5 patients induced to AF while 2 served as controls. The results indicated that, in order to find a statistically significant difference, there was a need to include 28

\section{Strengths and limitations of this study}

- Large sample size compared to other invasive studies.

- Prototype research concerning neurohormones as well the induction of atrial fibrillation.

- A broad view presented of the symptoms and mechanisms of atrial fibrillation by studying the relationship between symptom burden and metabolic aspects, atrial function, different neurohormones as well as the effect of radiofrequency ablation.

- Only patients considered for catheter ablation are included in the study.

patients; for safety reasons, 45 patients will be included.

Ethics and dissemination: The SMURF study is approved by the Regional Ethical Review Board at the Faculty of Health Sciences, Linköping, Sweden. The results will be presented through peer-review journals and conference presentation.

Trial registration number: NCT01553045;

Pre-results.

\section{INTRODUCTION}

Symptom burden, Metabolic profile, Ultrasound findings, Rhythm, neurohormonal activation, haemodynamics and healthrelated quality of life (HRQoL) in patients with atrial Fibrillation (SMURF) takes a broad view of symptoms and mechanisms of 
atrial fibrillation (AF). In order to achieve this, we studied the quality of life, several neurohormones, metabolic aspects and atrial function in a randomised, controlled substudy where AF is induced: the 'interventional SMURF-study' was carried out.

$\mathrm{AF}$ is the most common cardiac arrhythmia with an estimated prevalence of $1.5-2 \%$ in the general population. However, a recent trial reports a considerably higher estimation of at least $2.9 \%$ in Swedish population. $^{12}$

$\mathrm{AF}$ is an independent risk factor for ischaemic stroke and is estimated to cause about $20-25 \%$ of all cases. ${ }^{34}$ It is also associated with an increased risk of heart failure ${ }^{5}$ and death. ${ }^{6}$ Several clinical risk factors, ECG and echocardiographic features and biochemical makers are associated with an increased risk of $\mathrm{AF}^{7-9}$ Patients with $\mathrm{AF}$ with no or minimal heart disease and no other known non-cardiac causes are considered as 'lone AF' cases.

$\mathrm{AF}$ has a great impact on HRQoL. ${ }^{10}{ }^{11}$ One major unresolved issue in $\mathrm{AF}$ is the large variation in symptoms. In some patients, AF is diagnosed accidentally during routine check-up, while in others, symptoms are severe and disabling. There are data supporting the contention that change in $\mathrm{AF}$ episode duration, ventricular rate and $\mathrm{AF}$ cycle length can predict symptoms in patients with paroxysmal AF (PAF). ${ }^{12}$ Moreover, there is evidence to show a relationship between perceived symptom burden and rhythm control, personality and gender. ${ }^{13-15}$

Obesity, and thus insulin resistance, is a major contributor to cardiovascular (CV) disorder and hence adds to the incidence of AF. ${ }^{16}{ }^{17}$ Insulin resistance as a part of the metabolic syndrome is also linked to diastolic ventricular dysfunction ${ }^{18}$ and might contribute to more pronounced symptoms of $\mathrm{AF}^{19}$ Thus, it is of interest to measure markers of insulin resistance such as obesity levels, fasting insulin, glucose and cholesterol in patients with $\mathrm{AF}$.

Acute alcohol intake increases the risk of arrhythmias, ${ }^{20}$ but moderate intake of alcohol appears to harbour protective effects against $\mathrm{CV}$ and also other metabolic disorders. ${ }^{21}$ High long-term intake of alcohol has been associated with an increased prevalence of $\mathrm{AF}^{22}$ but estimation of alcohol intake from questionnaires is affected by different sources of bias. Moreover, long-term intake of alcohol can be estimated by measuring ethyl glucuronide (EtG) in human hair. $^{23}$

Left atrial (LA) remodelling and enlargement is a powerful predictor of several CV events including stroke and death. ${ }^{24}$ Using the area-length method from the apical 4 and 2 chamber views, it is possible to measure LA volumes (LAVs) in each phase of the atrial cycle and, furthermore, to quantify LA function by generating ejection fractions (EF) for each cycle. Two-dimensional speckle tracking (2D-SpTr) echocardiography is a relatively novel method for quantifying myocardial wall deformation using tracking of acoustic speckles. ${ }^{25}$ It allows simultaneous analysis of radial and longitudinal myocardial deformation independent of insonation angle by automatically tracking myocardial segments and is validated against sonomicrometry. ${ }^{26}$ This method may provide a comprehensive assessment of LA function. ${ }^{27}$

AF is associated with the activation of several neurohormonal systems and, for some, the underlying activation mechanisms and their subsequent effects are unknown. The endocrine function of the heart is complex. B-type natriuretic peptide (BNP) and atrial natriuretic peptide (ANP) are examples of peptides involved in the cardiac endocrine. ${ }^{28}$ Natriuretic peptides are mainly produced by atrial myocytes in normal participants. ${ }^{29}$ As the heart is failing, an increased part of the production of ANP and BNP stems from the ventricular myocytes. Both peptides are known to be produced in response to the increased wall tension and in $\mathrm{AF}$, in order to restore and maintain $\mathrm{CV}$ homoeostasis. ${ }^{29-32}$ Elevated concentrations of these peptides have been reported in patients with AF without overt heart failure, ${ }^{33}$ and these lead to natriuresis and vasodilation in order to normalise haemodynamics. However, it has been reported that prolonged $\mathrm{AF}$ is associated with a reduced capacity by the atria to produce natriuretic peptides. ${ }^{34}$ The plasma concentrations of the natriuretic peptides are elevated in patients with $\mathrm{AF}$ and are known to decrease after conversion to sinus rhythm (SR)..$^{35}$ $\mathrm{BNP}$ and its N-terminal fragment of proBNP are not only used in risk assessment of patients with heart failure but also in those with $\mathrm{AF}^{36}$ Owing to its instability, ANP has not been used in clinical routine. A new, more stable method for quantification of the midregional fragment of the $\mathrm{N}$-terminal precursor of proANP (MR-proANP) has been introduced, ${ }^{37}$ and may be useful in risk stratification of patients with AF. Vasopressin (AVP), a non-cardiac plasma marker of CV disease, is released from the neurohypophysis in response to changes in plasma osmolality, and is involved in osmoregulation and CV homoeostasis. The plasma concentration of AVP is increased in patients with heart failure and is associated with left ventricular dysfunction. ${ }^{38}$ However, AVP, as such, is not a clinically useful plasma marker because it rapidly degrades in the circulation. Instead, copeptin, the C-terminal fragment of proAVP, has emerged as a promising surrogate biomarker for the measurement of AVP concentration. ${ }^{39}$ Adrenomedullin (ADM) originates primarily in adrenal medulla and endothelial cells where cellular stress, ischaemia and hypoxia induce increased production. ${ }^{40}$ It is capable of promoting vasorelaxation, natriuresis and diuresis, thus contributing to maintaining circulatory homoeostasis. ${ }^{41}$ The mid-regional portion of pro-ADM (MR-proADM) is more stable than ADM and therefore better suited for clinical practice and assessment in stored samples. ${ }^{42}$ The reaction to AF initiation is unknown. Moreover, the role of $\mathrm{ADM}$ and AVP-hormonal system has not been studied in patients with AF. 


\section{AIMS}

The aims of this study are: (1) to correlate the variety in symptom burden in patients with AF eligible for radiofrequency ablation (RFA) as measured by patientreported outcomes: HRQoL, perceived control over the heart disease, anxiety and depression with objective indicators such as peptide levels, echocardiographic data, haemodynamics and metabolic profile; (2) to study the short-term effects of successful RFA peptides, LA function and patient symptoms; (3) to study the level of different peptides in peripheral vein blood and two sites of the heart (coronary sinus (CS) and LA) and to give an insight into the neurohormonal reaction after RFA in patients with AF; (4) to echocardiographically quantify LA function and intracardiac pressures in patients with $\mathrm{AF}$; and (5) to analyse relationships of components of the metabolic syndrome with increased risk for recurrence of $\mathrm{AF}$ and increased $\mathrm{LAV}$.

Furthermore, in order to study possible neurohormonal and intracardiac pressure changes directly after initiation of $\mathrm{AF}$, an interventional study is to be performed.

\section{METHODS}

\section{Study design}

This is an observational study in a single-centre cohort. A group of patients will also be included in an interventional randomised substudy. The first patient was included in January 2012. A total of 200 patients are planned to be included into the main study and 45 patients into the interventional study with a follow-up period of 12 months. No participants will be recruited into the randomised section of the study until prior to registration at http://www.clinicaltrials.gov. Enrolment is expected to last approximately 2.5 years and the study is to be completed by the end of 2015 .

\section{Study population}

Patients eligible for study participation are those referred for RFA of AF for the first time at the University Hospital in Linköping, Sweden. Consecutive patients are asked about study participation and are included if they meet the inclusion and do not have any exclusion criteria. Inclusion and exclusion criteria are presented in table 1.

Patients eligible for the main study, who are free from arrhythmia during 4 days prior to RFA, can be included in the interventional study. The recruitment process and follow-up is presented in figure 1 .

\section{Informed consent and ethical considerations}

All patients gave written informed consent, and the study participation is documented in the patient's medical record. Informed consent will be obtained by medical doctors participating in the study. The study complies with the Declaration of Helsinki. ${ }^{43}$ The study is to be periodically externally monitored in order to identify potential sources of bias. Any changes of study
Table 1 Inclusion and exclusion criteria in the SMURF main study
Inclusion criteria

Patients $>17$ years of age with paroxysmal or the Linköping University Hospital for the first RFA for AF

Informed consent persistent AF, referred to

\section{Exclusion criteria}

Previous catheter or surgical AF ablation

Ejection fraction $<35 \%$

Previous or expected heart surgery

Ongoing or previous acute coronary syndrome during the last 3 months before ablation

Poor understanding of the Swedish language, or other inabilities to independently fill out the questionnaires

$\mathrm{AF}$, atrial fibrillation; RFA, radiofrequency ablation. objectives, protocol and population, will require a formal amendment to the protocol. All study-related information is stored securely in a local database. All laboratory specimens and other data will be marked by a coded identification number. Further use of stored blood samples will require renewed evaluation by the Regional Ethical Review Board at the Faculty of Health Sciences, Linköping, Sweden. All recorded personal identifiers will be stored in the investigator site file and identified by code number. All investigators will be given access to a cleaned data set. All data sets will be password protected. To ensure confidentiality, data dispersed to project team members will be blinded of any identifying participant information. The results of the trial will be presented at regional and international congresses, and in peer-reviewed journals, and will also be released to the participating physicians, referring physicians and patients.

\section{Questionnaires}

Patient-reported outcome measures (PROMs) are assessed with four questionnaires, at the start of the study (baseline measurement) and after 4 and 12 months. The following questionnaires are used.

\section{Medical outcomes study 36-item short-form health survey}

36-Item short-form health survey (SF-36) is a generic questionnaire designed to measure an individual's physical and mental health (MH). It comprises 35 items grouped into eight scales and one question concerning changes in health outside the scales. The eight scales represent physical functioning, role limitations due to physical health problems, bodily pain, general health, vitality (energy/fatigue), social functioning, role limitations due to emotional problems (RE) and MH (psychological distress and psychological well-being). The eight scales are summarised in two dimensions; physical and 
Figure 1 Enrolment and follow-up flow chart $(A F$, atrial fibrillation; HRQoL, health-related quality of life;RFA, radiofrequency ablation).

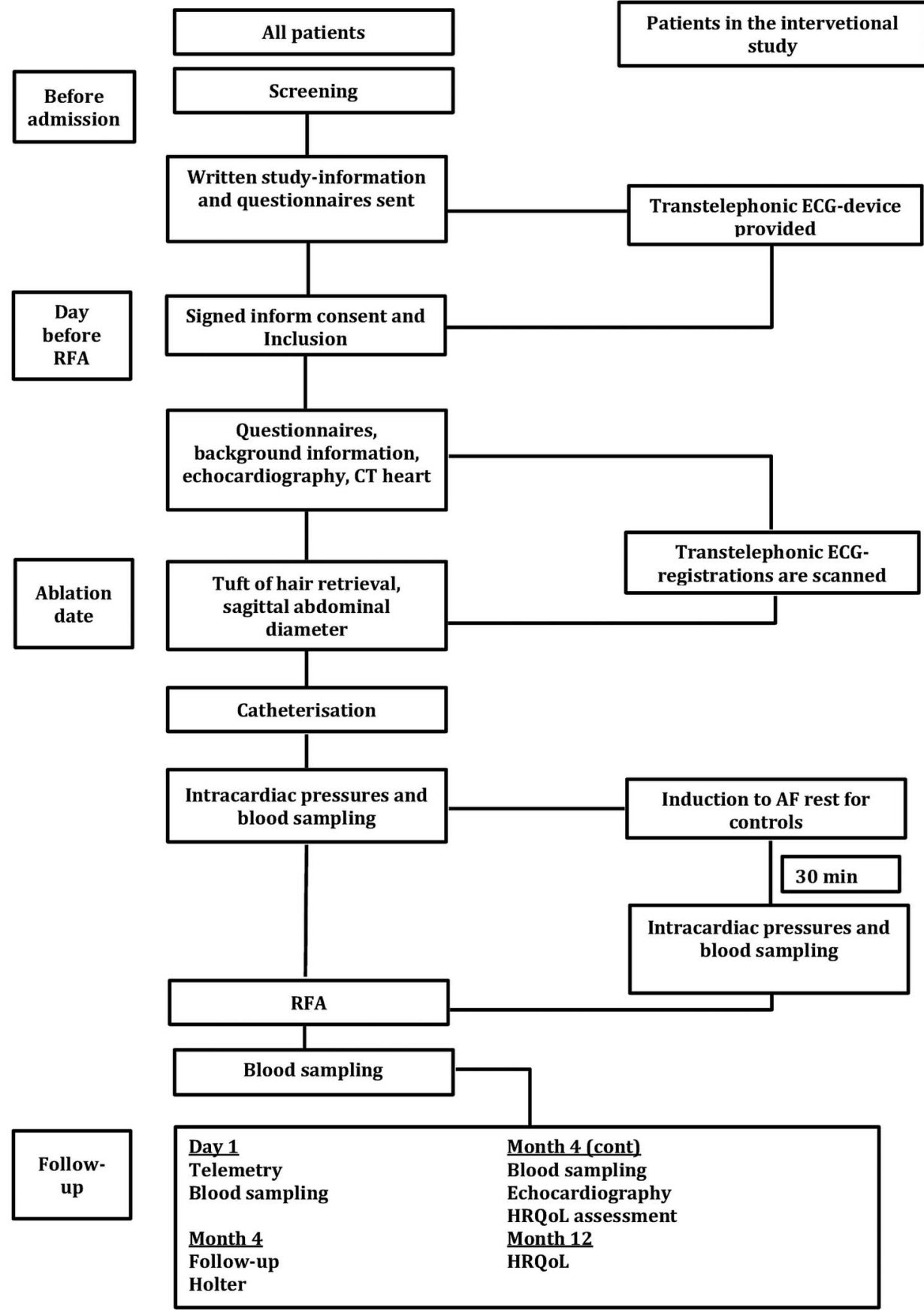

mental component summary. For each of the eight scales and the two summary dimensions, scores are coded, summed and transformed to a scale from 0 (worst possible health) to 100 (best possible health). The scoring of the SF-36 data is carried out as described by Ware and Sherbourne, ${ }^{44}$ and Ware et $a l^{45}$ SF-36 has been widely used in research including in studies on patients with arrhythmias. ${ }^{46}$

\section{Arrhythmia-specific questionnaire in tachycardia and} arrhythmia

The disease-specific arrhythmia-specific questionnaire in tachycardia and arrhythmia (ASTA) questionnaire is divided into three separate parts, where ASTA part I evaluates the patient's latest episode of arrhythmia, pharmacological treatment and-important at follow-up -one question concerning the existence of any arrhythmia. ASTA part II covers symptom burden: the ASTA symptom scale has nine items and a four-point response scale with response alternatives from 0 to 3 : 'No (0); Yes, to a certain extent (1); Yes, quite a lot (2) or Yes, a lot (3)'. A summarised score is calculated for the items, a higher score implies higher symptom burden due to the heart rhythm disturbance. Outside of the symptom scale there are questions concerning frequency of arrhythmia, duration and palpitations, and if there are factors influencing arrhythmia occurrence and the experience of near syncope and/or syncope in connection with 
arrhythmia with the response alternatives 'No or Yes'. ASTA part III is on HRQoL: the ASTA HRQoL scale has 13 items and describes the influence of arrhythmia on daily life. The response alternatives are the same as for the ASTA symptom scale (please see above) where higher scores reflect a worse effect on HRQoL. ${ }^{47} 48$

\section{Hospital anxiety and depression scale}

The domain-specific hospital anxiety and depression scale (HADS) questionnaire is used to evaluate symptoms of anxiety and depression. The questionnaire consists of two sections, where anxiety is assessed with seven questions (HADS-A) and depression with the remaining seven (HADS-D). Responses are scored on a scale of 03 ; with higher scores denoting more psychological distress. The score for each subscale (anxiety and depression) can range from 0 as lowest to a maximum score of 21 . The scores are categorised as normal $(0-7)$, mild (8$10)$, moderate (11-14) and severe (15-21) anxiety and/ or depression, respectively. ${ }^{49} 50$

\section{Control attitudes scale}

The control attitudes scale (CAS) is developed to measure perceived control among patients with cardiac diseases. In this paper, the validated Swedish version is used. The scale consists of four items, two about perceived control and two about helplessness. Furthermore, two of the items reflect the patient's own perceptions and two reflect the patients' perception about family members' perceived control. Patients rate each item on a seven-point Likert-type scale, from 'Not at all' to 'Very much'. Two of the item scores are reversed and then a total score is calculated by summing the responses. The total score can vary between 4 and 28 , where higher scores indicate greater perceived control. ${ }^{51} 52$

\section{Interviews}

A subset of patients will be included in an interview study exploring the patients' daily life situation due to $\mathrm{AF}$. An interview-guide including semistructured questions is constructed. All interviews are conducted by one researcher and begin with an initial open-ended question followed by questions intended to guide, refocus or clarify the respondent's answer. The interviews will be made before RFA, and will be audio recorded with the patients' permission. Qualitative inductive content analysis (conventional content analysis) is chosen to interpret the meaning from the content of text data by classifying the data into codes and categories. The data will be coded aided by the software system NVivo9 (QSR International Pty Ltd, Melbourne, Australia).

\section{Brief intermittent transtelephonic ECG}

The ECG system used has been developed by Zenicor $\mathrm{AB}$ and consists of a device that can register a short ECG sequence $(30 \mathrm{~s})$ by having participants place their thumbs on two measuring plates. The method has been validated $^{53}$ and used in order to improve screening of silent $\mathrm{AF}$ in patients with ischaemic stroke ${ }^{54}$ and as an event-recorder.

A registration is sent by telephone to a central database, which can only be accessed by authorised personnel. Rhythm analysis is performed prior to randomisation on patients who are eligible for the interventional study. The rhythm is classified into the following categories: SR, $\mathrm{AF}$, atrial tachycardia or 'not specified rhythm'.

\section{Echocardiography}

Before RFA, all patients undergo a transthoracic and a transesophageal echocardiographic examination (TTE and TEE, respectively) using the GE Vivid 7 or Vivid E9 system (GE Healthcare, Horten, Norway) with a $3.5 \mathrm{MHz}$ transducer for TTE and a $7 \mathrm{MHz}$ transducer for TEE. Measurements and evaluations are performed according to the guidelines of European Society of Echocardiography. ${ }^{55}$ Blood pressure is recorded and heart rate monitored during the examination. All data are stored digitally for further offline analysis (Echo PAC version BT 12, GE Healthcare, Horten, Norway).

\section{TTE measurements}

Left ventricular EF is calculated by biplane Simpson's method. LAV is measured using the biplane area-length method and is corrected for body surface area to obtain LAV index (LAVI). Left ventricular filling velocities (E and A) are obtained by pulsed-wave Doppler in the apical four-chamber $(4 \mathrm{CH})$ view. The ratio of E/A velocity is measured. Tissue Doppler images in the $4 \mathrm{CH}$ view are obtained and used offline in order to measure the early diastolic velocity $\left(E^{\prime}\right)$ in the mitral septal annulus. $\mathrm{E} / \mathrm{E}^{\prime}$ ratio is then calculated.

\section{Transesophageal echocardiography}

Patients undergo TEE examination under pharyngeal anaesthesia. The LA appendage (LAA) is visualised and examined thoroughly for the possible presence of thrombus. Filling and emptying LAA flow velocities are assessed by pulsed wave Doppler. The sample area is positioned at the outlet of LAA, $1 \mathrm{~cm}$ away from the LA cavity.

\section{Speckle tracking echocardiography}

2D echocardiographic images of the LA are obtained from the apical $4 \mathrm{CH}$ and $2 \mathrm{CH}$ view for speckle tracking evaluation. All images are obtained with a stable ECG recording. Three consecutive beats are stored in a cine loop format. The frame rate is adjusted between 55 and $100 \mathrm{frames} / \mathrm{s}$. The endocardial border of the LA is manually traced. A region of interest (ROI) is automatically generated by the software (Echo PAC version BT 12, GE Healthcare, Horten, Norway); manual adjustments are performed if necessary. Subsequently, the ROI is divided into six segments and if these are of acceptable quality then the software is allowed by the user to perform further analysis. Segments of poor quality are either automatically or manually rejected from further processing. Finally, strain and strain rate curves are 
generated. Calculations by averaging the values observed in all LA segments are made for the measurement of peak atrial longitudinal strain, peak atrial contraction strain, time to peak longitudinal strain, time to peak contraction strain, LA strain rate during ventricular systole (LA-SRs), strain rate during ventricular passive filling (LA-SRE) and strain rate during atrial active contraction (LA-SRA). If some segments are excluded from the analysis, the calculation is to be made by averaging the remaining segments.

\section{Ablation procedure}

Procedures are performed under conscious sedation using propofol and remifentanil. Two trans-septal sheaths are inserted through the right femoral vein into the LA and perfused using heparinised saline with irrigation rate of $3 \mathrm{~mL} / \mathrm{h}$. Heparin is administered to maintain an activated clotting time of $>350 \mathrm{~s}$ throughout the procedure. RFA is performed under the guidance of a computer-based mapping system, CARTO (Biosense Webster, Diamond Bar, California, USA). Mapping and ablation are performed using an open-irrigated catheter (ThermoCool, Biosense Webster, Diamond Bar, California, USA). A 7-F, 20-pole circumferential diagnostic catheter is used for the assessment of pulmonary vein activation and isolation (Lasso, Biosense Webster, Diamond Bar, California, USA). Radiofrequency energy is delivered in a power-controlled mode with maximum energy setting of $35 \mathrm{~W}$ at an irrigation rate of $17-30 \mathrm{~mL} /$ min, and the maximum energy setting in the posterior wall is $25 \mathrm{~W}$. The end point of the procedure is electrical disconnection of all pulmonary veins by antral ablation verified during SR by entry and exit block of all pulmonary veins, that is, DC conversion is performed if needed prior to the end of the procedure. In patients with persistent $\mathrm{AF}$, additional ablation in order to create LA lines is at the discretion of the operator and verified by pacing manoeuvres.

\section{Pressure measurements}

The sagittal thoracic diameter is measured in the second intercostal space and the reference pressure (zero level) is placed in the middle of this diameter. A-wave, v-wave and mean pressures are measured in the right atrium (RA), LA and systolic and diastolic pressures in the right ventricle (RV) using the multipurpose (MR A1) high flow (Cordis) 5F catheter, during quiet breathing, and recorded at least for $15 \mathrm{~s}$ for offline analysis (EP-WorkMate; St Jude Medical, Saint Paul, Minnesota, USA).

\section{Markers of metabolic syndrome and measurement of long-term alcohol intake}

The laboratory analyses are performed at the Department of Clinical Chemistry at Linköping University Hospital. Levels of cholesterol, high-density lipoprotein (HDL) and triglycerides are measured using enzymatic methodology and spectrophotometry
(Selectra E, Vital Scientific, the Netherlands/Triolab). Low-density lipoprotein (LDL) is calculated by Friedewalds formula: $\mathrm{LDL}=$ cholesterol $-\mathrm{HDL}-0.45 \times \mathrm{fS}$ / P-triglycerides. Glycated haemoglobin (HbA1c) is analysed according to the Swedish Mono-S high performance liquid chromatography (HPLC) method, and all HbAlc values are converted to diabetes control and complications trial (DCCT) standard values using the formula: HbA1c DCCT $=0.923 \times \mathrm{HbA1c} \quad($ Mono-S $)+1.345$ $\left(\mathrm{R}^{2}=0.998\right)$ and are to be reported in international federation of clinical chemistry and laboratory medicine (IFCC) units. Levels of apolipoprotein B (apoB) and apolipoprotein A1 (apoA1) are measured by immunoturbidimetric assays (Advia 1800, DakoCytomation, Glostrup, Denmark).

Hair samples are collected as close as possible above the skin and the proximal $2 \mathrm{~cm}$ are used for estimation of amount of EtG. Analyses are performed as published earlier. $^{23}$

\section{Cardiac biomarkers}

Blood samples are collected in plastic vials containing EDTA. The vials are centrifuged at $3100 \mathrm{~g}$ for $20 \mathrm{~min}$ and then frozen at $-70^{\circ} \mathrm{C}$. No sample is to be thawed more than twice.

Concentrations of proBNP 1-76 (NT-proBNP) are measured on the Elecsys 2010 platform (Roche Diagnostics, Mannheim, Germany). Plasma concentrations of MR-proADM and copeptin are measured on a kryptor platform (Brahms AG, Hennigsdorf, Germany) and MR-proANP in commercial assay (MR-proANP; Brahms AG, Hennigsdorf, Germany).

\section{Study's flow chart}

First contact

After screening, potentially eligible patients receive written information about the study and the following forms: SF-36, ASTA, HADS and CAS. Patients eligible for the interventional study are provided with a transtelephonic device and instructed to record and send ECG recordings twice daily and extra recordings in case of symptoms during the 4 days prior to RFA.

\section{Baseline evaluation}

Prior to RFA patients receive further oral information and informed consent is signed. A full baseline evaluation, including medical history, physical examination and a 12-lead ECG, is performed. All patients undergo TTE, TEE and a CT scan of the heart according to the clinical routine.

All registered transtelephonic ECGs sent from patients eligible for the interventional study are screened, and if free from arrhythmias, the respective patient can be included, otherwise the patient continues in the main study (figure 2).

The sagittal abdominal diameter is measured and a tuft of hair is cut for the analysis of EtG. 


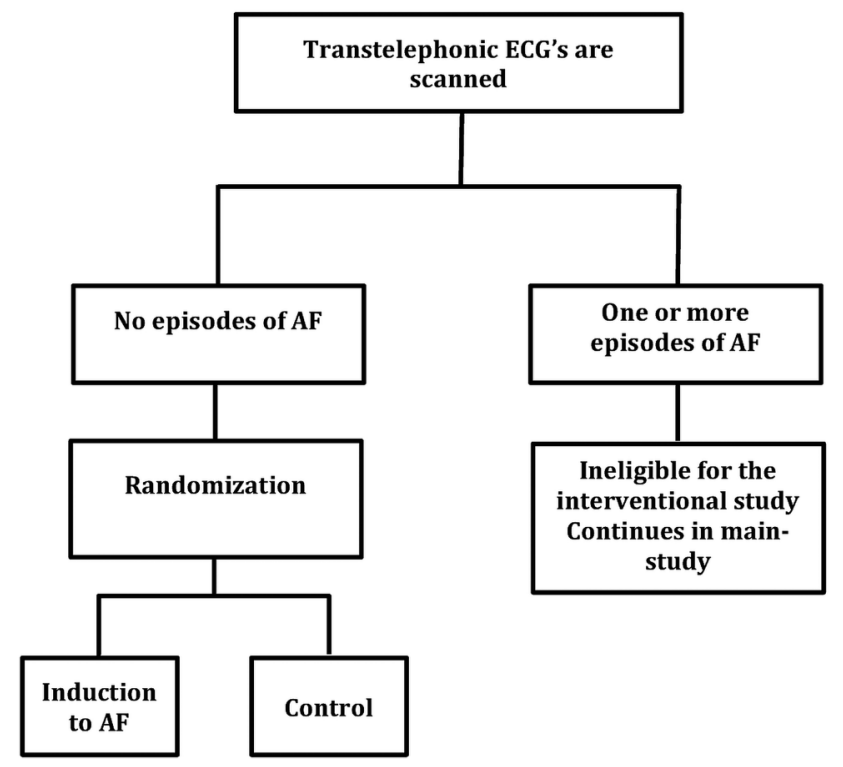

Figure 2 Eligibility and randomisation process in the interventional study (AF, atrial fibrillation).

All patients are then catheterised according to clinical routine and blood samples (glucose, HbAlc, insulin, blood status, aspartate aminotransferase, alanine aminotransferase, lactate dehydrogenase, bilirubin, cholesterol levels including LDL, HDL, lipoproteins A and B, creatinine, cystatin $\mathrm{C}$, thyroid-stimulating hormone, $\mathrm{T}_{4}, \mathrm{~T}_{3}$ and high-sensitivity $\mathrm{G}$ reactive protein) are obtained from the femoral vein. Blood samples for the analysis of NT-proBNP, MR-proANP, copeptin and MR-proADM are drawn from the femoral vein, CS and LA (baseline sampling) using a multipurpose (MR A1) high-flow (Cordis) catheter. Intracardiac pressures are recorded in the RA, LA and RV.

\section{Interventional study: induced AF}

After baseline blood sample retrieval and intracardiac pressure measurements, patients randomised to the active group are induced to AF with burst pacing from CS (CL: 170-300 ms). AF is maintained for $30 \mathrm{~min}$ and immediately re-induced when necessary. Blood samples for analysis of biomarkers are drawn in the same way as before induction.

Patients randomised to the control group are only monitored for $30 \mathrm{~min}$ after baseline blood sampling. Intracardiac pressures are then recorded and blood samples drawn in the same way as mentioned earlier (figures 1 and 2).

Thereafter, RFA is performed regardless of the patient's actual rhythm. At the end of the procedure, blood samples are drawn from the femoral vein for peptide analysis.

\section{Follow-up}

The day after RFA, blood samples are drawn from a peripheral vein for peptide analyses. At 4-month follow-up, a
$24 \mathrm{~h}$ Holter, blood sampling and assessment with the previously used questionnaires are performed. TTE control is performed in a subgroup of patients who are residents of the Östergötland County. At 12-month follow-up, only assessment with questionnaires will be made.

\section{End points}

\section{Primary end point}

Primary end point is HRQoL and arrhythmia symptom burden in relation to the level of four different peptides: NT-proBNP, copeptin, MR-proADM and MR-proANP.

\section{Interventional study, primary end point}

Primary end point for the interventional study is changes in NT-proBNP, MR-proANP, MR-proADM and copeptin 30 min after the induction of AF compared to controls in three sample sites (femoral vein, LA and CS).

\section{Secondary end points}

- Reaction of NT-proBNP, MR-proANP, copeptin and MR-proADM directly after $18 \mathrm{~h}$ and 4 months after RFA in relation to heart rhythm at admission, heart rate and type of $\mathrm{AF}$.

- Measurements of a possible concentration of copeptin and MR-proANP indicating a possible cardiac production of the two biomarkers and the levels of NT-proBNP and MR-proANP in LA and in CS.

- Correlation between MR-proANP, NT-proBNP, copeptin and MR-proADM levels, and LA function assessed by $2 \mathrm{D}$-strain measurements.

- Echocardiographic description of LA function by the assessment of LAV, LA EF, 2D strain, and strain rate of the LA and LA appendix function in relation to heart rhythm, type of AF and long-term alcohol consumption.

- Correlation between estimated alcohol consumption, HRQoL and arrhythmia freedom after RFA.

- Correlation between the metabolic syndrome, sagittal abdomen diameter and AF recurrence after RFA as well as to LAV.

- HRQoL and symptom burden in patients with AF prior to, and 4 and 12 months after RFA.

- Peptide levels and LA function as predictors of arrhythmia-freedom 4 months after ablation.

- Effect of RFA on LA, LAA function and peptide levels 4 months after the ablation.

- Levels of NT-proBNP, MR-proANP, MR-proADM and copeptin in peripheral blood, LA and CS in relation to different types of $\mathrm{AF}$ and to $\mathrm{CHA}_{2} \mathrm{DS}_{2}$-VASc, and AF risk score.

- RA, RV and LA pressures in patients with AF eligible for RFA in relation to LA function and patients' symptom burden.

- Pressure changes in RA, RV and LA after induction of AF.

- A description of how a patient's daily life is influenced; exploring the relationship between arrhythmia-specific symptoms, HRQoL, anxiety and 
depression, perceived control and healthcare contacts, a qualitative study.

\section{Interventional study, secondary end points}

- Activation of neurohormonal systems and changes in intracardiac pressures after initiation of $\mathrm{AF}$ in patients with a high symptom burden.

- Correlation between peptide changes, and changes in recorded pressures and echocardiographic data.

\section{Randomisation concerning the interventional study}

Forty-five patients are to be randomised. Randomisation is performed with a 2:1 allocation ratio, that is, for every two patients who are randomised to AF induction, one is to be randomised to the control group.

Carefully prepared, sealed, opaque, identical and serially numbered envelopes produced by Linkoping's academic research centre are provided in order to ensure impartial randomisation. A trial investigator will assign participants to interventions. Owing to the nature of the interventional study, blinding of participants or staff was not possible.

\section{Statistical issues and sample size consideration}

Baseline characteristics will be reported as means and $\mathrm{SD}$ for parametric and median, and IQR for nonparametric data. Normality will be tested by Kolmogorov-Smirnov test with $\mathrm{p}<0.05$ to be considered as statistically significant. Continuous data that are not normally distributed will be transformed if needed. Categorical data will be presented as number and percentage. All tests performed will be two-sided and a $\mathrm{p}<0.05$ will be considered statistically significant.

Full statistical analysis is to be published separately when presenting each outcome. STROBE statement for the main study and CONSORT statement for the interventional study will be followed when publishing the results.

All missing values and those lost to follow-up will be excluded from the respective analysis. The analyses will be performed using SPSS V.22.0 (SPSS, Chicago, Illinois, USA). To the best of our knowledge, there have been no previous studies performed addressing our primary and several of the secondary end points, thus a power analysis was not possible. There have been studies on the reaction of ANP and BNP in patients with AF after RFA, and after successful electrical conversion to $\mathrm{SR}^{35}{ }^{56}$ in which 40-60 patients were included, in order to show significant results. We concluded that 200 patients would be sufficient. The interventional study was preceded by a small pilot study where five patients were induced into $\mathrm{AF}$ and two patients served as controls. The assumption of the possible difference on MR-proANP level was that as MR-proANP is preproduced and stored in cardiac myocyte granules, ${ }^{57}$ the initiation of $\mathrm{AF}$ could lead to a direct release of the peptide and higher levels of MR-proANP could be measured $30 \mathrm{~min}$ after the induction to AF. The results indicated that, in order to find a statistically significant difference between the intervention and the control group on the reaction of the levels of MR-proANP with a power of $90 \%$ and type I error of 0.05 , there was a need to include 28 patients. As there are other neurohormonal systems to be tested, we increased the number of participants to 45 for safety reasons. The sample size calculation was performed using a commercially available statistical analysis software package (STATISTICA 10; Statsoft, Dell Statistica, Tulsa, Oklahoma, USA). Unequal allocation ratio was taken into account.

\section{Author affiliations}

${ }^{1}$ Department of Cardiology and Department of Medical and Health Sciences, Linköping University, Linköping, Sweden

${ }^{2}$ Department of Medical and Health Sciences, Faculty of Health Sciences, Linköping University, Linköping, Sweden

${ }^{3}$ Department of Clinical Physiology and Department of Medical and Health Sciences, Linköping University, Linköping, Sweden

Contributors EC participated in all study aspects, design, pre-study administration, performing echocardiography and in laboratory analyses, drafted, and revised and approved this manuscript. UWparticipated in the study design, created the ASTA questionnaire for analysis of symptom burden and health-related quality of life, was responsible for analysing data on symptom burden and health-related quality of life, was responsible for the associated part of the manuscript, and revised and approved this manuscript. FNparticipated in the study design with special responsibility for data on the metabolic syndrome, and revised and approved this manuscript.

ENparticipated in the study design with special responsibility for data on echocardiography and intracardiac pressures, and revised and approved this manuscript. ASparticipated in the study design, was co-responsible, with UW, for analysis of symptom burden and health-related quality of life, and revised and approved this manuscript. UAparticipated in the study design with special responsibility for data on neurohormones, and revised and approved this manuscript. HWconceived the idea of the study, is the main investigator with overall responsibility for the study, and revised and finalised this manuscript.

Funding The SMURF study is supported by grants from ALF grants (County Council of Östergötland), the Carldavid Jönsson Research Foundation, the Heart Foundation, Linköping University, and by unrestricted grants from Biosense Webster, and Johnson and Johnson.

\section{Competing interests None declared.}

Ethics approval The Regional Ethical Review Board at the Faculty of Health Sciences, Linkoping, Sweden.

Provenance and peer review Not commissioned; externally peer reviewed.

Data sharing statement The results will be presented through peer-review journals and conference presentation. Additional publications are planned on this data from this institution.

Open Access This is an Open Access article distributed in accordance with the Creative Commons Attribution Non Commercial (CC BY-NC 4.0) license, which permits others to distribute, remix, adapt, build upon this work noncommercially, and license their derivative works on different terms, provided the original work is properly cited and the use is non-commercial. See: http:// creativecommons.org/licenses/by-nc/4.0/

\section{REFERENCES}

1. Camm AJ, Lip GY, De Caterina R, et al. 2012 Focused update of the ESC Guidelines for the management of atrial fibrillation: an update of the 2010 ESC Guidelines for the management of atrial fibrillation. Developed with the special contribution of the European Heart Rhythm Association. Eur Heart J 2012;33:2719-47.

2. Friberg L, Bergfeldt L. Atrial fibrillation prevalence revisited. J Intern Med 2013;274:461-8. 
3. Lin HJ, Wolf PA, Kelly-Hayes M, et al. Stroke severity in atrial fibrillation. The Framingham Study. Stroke 1996;27:1760-4.

4. Wolf PA, Abbott RD, Kannel WB. Atrial fibrillation as an independent risk factor for stroke: the Framingham Study. Stroke 1991;22:983-8.

5. Stewart S, Hart CL, Hole DJ, et al. A population-based study of the long-term risks associated with atrial fibrillation: 20-year follow-up of the Renfrew/Paisley study. Am J Med 2002;113:359-64.

6. Benjamin EJ, Wolf PA, D'Agostino RB, et al. Impact of atrial fibrillation on the risk of death: the Framingham Heart Study. Circulation 1998;98:946-52.

7. January CT, Wann LS, Alpert JS, et al. American College of Cardiology/American Heart Association Task Force on Practice Guidelines. 2014 AHA/ACC/HRS Guideline for the Management of Patients with Atrial Fibrillation: a report of the American College of Cardiology/American Heart Association Task Force on Practice Guidelines and the Heart Rhythm Society. J Am Coll Cardiol 2014;64:e1-76.

8. Kodama S, Saito K, Tanaka S, et al. Alcohol consumption and risk of atrial fibrillation: a meta-analysis. J Am Coll Cardiol 2011;57:427-36.

9. Frost L, Vestergaard P, Mosekilde L. Hyperthyroidism and risk of atrial fibrillation or flutter: a population-based study. Arch Intern Med 2004;164:1675-8.

10. Kang Y. Relation of atrial arrhythmia-related symptoms to health-related quality of life in patients with newly diagnosed atrial fibrillation: a community hospital-based cohort. Heart Lung 2006;35:170-7.

11. Thrall G, Lane D, Carroll D, et al. Quality of life in patients with atrial fibrillation: a systematic review. Am J Med 2006;119:448.e1-19.

12. Silberbauer J, Veasey RA, Cheek E, et al. Electrophysiological characteristics associated with symptoms in pacemaker patients with paroxysmal atrial fibrillation. J Interv Card Electrophysiol 2009;26:31-40.

13. Steg PG, Alam S, Chiang CE, et al. Symptoms, functional status and quality of life in patients with controlled and uncontrolled atrial fibrillation: data from the RealiseAF cross-sectional international registry. Heart 2012;98:195-201.

14. Paquette $M$, Roy $D$, Talajic M, et al. Role of gender and personality on quality-of-life impairment in intermittent atrial fibrillation. Am J Cardiol 2000;86:764-8.

15. Son YJ, Song EK. The impact of type D personality and high-sensitivity C-reactive protein on health-related quality of life in patients with atrial fibrillation. Eur J Cardiovasc Nurs 2012;11:304-12.

16. Vyssoulis G, Karpanou E, Adamopoulos D, et al. Metabolic syndrome and atrial fibrillation in patients with essential hypertension. Nutr Metab Cardiovasc Dis 2013;23:109-14.

17. Tanner RM, Baber U, Carson AP, et al. Association of the metabolic syndrome with atrial fibrillation among United States adults (from the REasons for Geographic and Racial Differences in Stroke [REGARDS] Study). Am J Cardiol 2011;108:227-32.

18. von Bibra H, St John Sutton M. Diastolic dysfunction in diabetes and the metabolic syndrome: promising potential for diagnosis and prognosis. Diabetologia 2010;53:1033-45.

19. Shigematsu $Y$, Hamada M, Nagai T, et al. Risk for atrial fibrillation in patients with hypertrophic cardiomyopathy: association with insulin resistance. J Cardiol 2011;58:18-25.

20. Rich EC, Siebold C, Campion B. Alcohol-related acute atrial fibrillation. A case-control study and review of 40 patients. Arch Intern Med 1985;145:830-3.

21. Corrao G, Rubbiati L, Bagnardi V, et al. Alcohol and coronary heart disease: a meta-analysis. Addiction 2000;95:1505-23.

22. Samokhvalov AV, Irving HM, Rehm J. Alcohol consumption as a risk factor for atrial fibrillation: a systematic review and meta-analysis. Eur J Cardiovasc Prev Rehabil 2010;17:706-12.

23. Kronstrand R, Brinkhagen L, Nyström FH. Ethyl glucuronide in human hair after daily consumption of 16 or $32 \mathrm{~g}$ of ethanol for 3 months. Forensic Sci Int 2012;215:51-5

24. Benjamin EJ, D'Agostino RB, Belanger AJ, et al. Left atrial size and the risk of stroke and death. The Framingham Heart Study. Circulation 1995;92:835-41.

25. Leitman M, Lysyansky P, Sidenko S, et al. Two-dimensional strain-a novel software for real-time quantitative echocardiographic assessment of myocardial function. J Am Soc Echocardiogr 2004;17:1021-9.

26. Langeland S, D'Hooge J, Wouters PF, et al. Experimental validation of a new ultrasound method for the simultaneous assessment of radial and longitudinal myocardial deformation independent of insonation angle. Circulation 2005;112:2157-62.

27. Vianna-Pinton $\mathrm{R}$, Moreno CA, Baxter CM, et al. Two-dimensional speckle-tracking echocardiography of the left atrium: feasibility and regional contraction and relaxation differences in normal subjects. J Am Soc Echocardiogr 2009;22:299-305.

28. Flynn TG, de Bold ML, de Bold AJ. The amino acid sequence of an atrial peptide with potent diuretic and natriuretic properties. Biochem Biophys Res Commun 1983;117:859-65.

29. Goetze JP, Friis-Hansen L, Rehfeld JF, et al. Atrial secretion of B-type natriuretic peptide. Eur Heart J 2006;27:1648-50.

30. Inoue S, Murakami Y, Sano K, et al. Atrium as a source of brain natriuretic polypeptide in patients with atrial fibrillation. J Card Fail 2000;6:92-6.

31. Yasue $\mathrm{H}$, Yoshimura $\mathrm{M}$, Sumida $\mathrm{H}$, et al. Localization and mechanism of secretion of B-type natriuretic peptide in comparison with those of A-type natriuretic peptide in normal subjects and patients with heart failure. Circulation 1994;90:195-203.

32. Clerico A, Emdin M. Diagnostic accuracy and prognostic relevance of the measurement of cardiac natriuretic peptides: a review. Clin Chem 2004;50:33-50.

33. Ellinor PT, Low AF, Patton KK, et al. Discordant atrial natriuretic peptide and brain natriuretic peptide levels in lone atrial fibrillation. J Am Coll Cardiol 2005;45:82-6.

34. van den Berg MP, Tjeerdsma G, Jan de Kam P, et al. Longstanding atrial fibrillation causes depletion of atrial natriuretic peptide in patients with advanced congestive heart failure. Eur J Heart Fail 2002;4:255-62.

35. Wozakowska-Kaplon B. Effect of sinus rhythm restoration on plasma brain natriuretic peptide in patients with atrial fibrillation Am J Cardiol 2004;93:1555-8.

36. Hijazi Z, Wallentin L, Siegbahn A, et al. N-terminal pro-B-type natriuretic peptide for risk assessment in patients with atrial fibrillation: insights from the ARISTOTLE Trial (Apixaban for the Prevention of Stroke in Subjects With Atrial Fibrillation). J Am Coll Cardiol 2013;61:2274-84.

37. Morgenthaler NG, Struck J, Thomas B, et al. Immunoluminometric assay for the midregion of pro-atrial natriuretic peptide in human plasma. Clin Chem 2004;50:234-6.

38. Chatterjee K. Neurohormonal activation in congestive heart failure and the role of vasopressin. Am J Cardiol 2005;95:8B-13B.

39. Voors $A A$, von Haehling $S$, Anker SD, et al. C-terminal provasopressin (copeptin) is a strong prognostic marker in patients with heart failure after an acute myocardial infarction: results from the OPTIMAAL study. Eur Heart J 2009;30:1187-94.

40. Sugo S, Minamino N, Kangawa K, et al. Endothelial cells actively synthesize and secrete adrenomedullin. Biochem Biophys Res Commun 1994;201:1160-6.

41. Bunton DC, Petrie MC, Hillier C, et al. The clinical relevance of adrenomedullin: a promising profile? Pharmacol Ther 2004;103:179-201.

42. Morgenthaler NG, Struck J, Alonso C, et al. Measurement of midregional proadrenomedullin in plasma with an immunoluminometric assay. Clin Chem 2005;51:1823-9.

43. [No authors listed]. [The Helsinki Declaration of the World Medical Association (WMA). Ethical principles of medical research involving human subjects]. Pol Merkur Lekarski 2014;36:298-301.

44. Ware JE Jr, Sherbourne CD. The MOS 36-item short-form health survey (SF-36). I. Conceptual framework and item selection. Med Care 1992;30:473-83.

45. Ware JE Jr, McCrady SK, Kosinski M, et al. SF-36 health survey manual and interpretation guide. New England Medical Center, 1993.

46. Walfridsson $\mathrm{U}$, Walfridsson $\mathrm{H}$, Arestedt $\mathrm{K}$, et al. Impact of radiofrequency ablation on health-related quality of life in patients with paroxysmal supraventricular tachycardia compared with a norm population one year after treatment. Heart Lung 2011;40:405-11.

47. Walfridsson U, Arestedt K, Stromberg A. Development and validation of a new Arrhythmia-Specific questionnaire in Tachycardia and Arrhythmia (ASTA) with focus on symptom burden. Health Qual Life Outcomes 2012;10:44.

48. Walfridsson U, Strömberg A, Årestedt K. Development and validation of an arrhythmia-specific scale in tachycardia and arrhythmia with focus on health-related quality of life. J Cardiovascular Nurs 2015;30:98-108.

49. Zigmond AS, Snaith RP. The Hospital Anxiety and Depression Scale. Acta Psychiatr Scand 1983;67:361-70.

50. Bjelland I, Dahl AA, Haug TT, et al. The validity of the Hospital Anxiety and Depression Scale. An updated literature review. J Psychosom Res 2002;52:69-77.

51. Moser DK, Dracup K. Psychosocial recovery from a cardiac event: the influence of perceived control. Heart Lung 1995;24:273-80.

52. Årestedt $\mathrm{K}$, Ågren S, Flemme I, et al. A psychometric evaluation of the four-item version of the Control Attitudes Scale for patients with cardiac disease and their partners. Eur J Cardiovasc Nurs 2015;14:317-25. 
53. Hendrikx T, Rosenqvist M, Wester P, et al. Intermittent short ECG recording is more effective than 24-hour Holter ECG in detection of arrhythmias. BMC Cardiovasc Disord 2014;14:41.

54. Doliwa Sobocinski P, Anggårdh Rooth E, Frykman Kull V, et al. Improved screening for silent atrial fibrillation after ischaemic stroke. Europace 2012;14:1112-16.

55. Lang RM, Badano LP, Mor-Avi V, et al. Recommendations for cardiac chamber quantification by echocardiography in adults: an update from the American Society of Echocardiography and the European Association of Cardiovascular Imaging. Eur Heart $J$ Cardiovasc Imaging 2015;16:233-71.

56. Sacher F, Corcuff JB, Schraub P, et al. Chronic atrial fibrillation ablation impact on endocrine and mechanical cardiac functions. Eur Heart J 2008;29:1290-5.

57. Dietz JR. Mechanisms of atrial natriuretic peptide secretion from the atrium. Cardiovasc Res 2005;68:8-17. 